\title{
ENGINEERING STUDIES ON JOINT BAR INTEGRITY, PART I: FIELD SURVEYS AND OBSERVED FAILURE MODES
}

\author{
David Y. Jeong \\ Volpe National Transportation Systems Center \\ US Department of Transportation \\ Cambridge, MA, USA
}

\author{
Radim Bruzek \\ ENSCO, Inc. \\ Applied Technology and Engineering Division \\ Springfield, VA, USA
}

\author{
Ali Tajaddini \\ Federal Railroad Administration \\ US Department of Transportation \\ Washington, DC, USA
}

\begin{abstract}
This paper is the first of a two-part series describing a research project, sponsored by the Federal Railroad Administration (FRA), to study the structural integrity of joint bars. In Part I of this series, observations from field surveys conducted on revenue service track are presented. Automated and visual inspections of rail joints were conducted to identify defective joint bars. Detailed information and measurements were collected at various joint locations.

The survey team consisted of personnel from ENSCO, Inc. and Transportation Technology Center, Inc. (TTCI), working in cooperation with staff from participating railroads. Part II of this series describes the development of finite element analyses of jointed rail, which is being carried out by the Volpe National Transportation Systems Center (Volpe Center).
\end{abstract}

\section{INTRODUCTION}

FRA has sponsored and managed research on safety matters related to railroad track and equipment for several decades [1]. Rail integrity is an area of research under the FRA Track Safety Research Program, which deals with the prevention and control of rail failures. Rail failures, or broken rails, usually originate from defects that form and grow in the rail head as a result of metal fatigue. Past rail integrity research focused on defects that occur in continuous welded rail (CWR), primarily because of an increasing trend in the railroad industry to replace bolted joint rail with CWR.

Rail joints, however, cannot be completely eliminated. For example, bolted joints are often used to connect strings of CWR. Bolted joints are also used for temporary repairs, and in sharp curves in which rapid wear may require frequent rail replacement. In addition, insulated rail joints are used to isolate the electric current for signaling purposes, which provides a means to detect broken rails when the circuit is interrupted.

In terms of mechanical performance, rail joints are considered as a weak link because the section properties (i.e. cross-sectional area and area moment of inertia) of the bars are typically less than those for the rail itself. Consequently, larger stresses and deflections are expected to occur at rail joints compared to the continuous rail under the same loads.

Figure 1 shows the number of incidents involving joint failures over the twenty-year period between 1993 and 2012. The source of the data is the FRA Rail Accident/Incident Reporting System (RAIRS) [2]. There are six cause codes in RAIRS for joint failure-related incidents: T201 - bolt-hole crack, T213 - compromise bar, T214 - insulated joint bar, T215 - non-insulated joint bar, T216 - broken or missing bolts, and T219 - rail defect with joint bar repair. Figure 1 also shows the total number of joint failures (i.e. summation of the incidents for each of the six cause codes) for each year during the time period.

RAIRS data shown in Figure 1 indicate that the incidents involving joint failures are very rare events. However, train derailments and collisions can result in severe consequences. Moreover, the structural integrity of rail joints has come under scrutiny because joint failures have been involved in accidents which led the release of hazardous materials in some cases and the fatalities and injuries to passenger in others:

- On May 27, 2000, 33 of 113 cars derailed near Eunice, Louisiana. The derailment resulted in a release of 
hazardous materials with explosions and fires. About 3,500 people were evacuated from the surrounding area. An investigation conducted by the National Transportation Safety Board (NTSB) determined that the probable cause was the failure of a set of joint bars that had remained in service with undetected defects [3].

- On January 18, 2002, 31 of 112 cars derailed near Minot, North Dakota. Several tank cars released over 200,000 gallons of anhydrous ammonia, which resulted in one fatality, over 300 injuries, and affected over 15,000 local residents. Broken joint bars and undetected defects were involved in this derailment [4].

- On October 16, 2004, three locomotives and 11 cars derailed near Pico Rivera, California. An estimated 5,000 gallons of diesel fuel were released from the locomotive fuel tanks when they ruptured as a result of the derailment. The NTSB determined that the probable cause of the derailment was the failure of a pair of insulated joint bars due to fatigue cracking [5].

- On October 10, 2007, 31 of 114 cars derailed in Painesville, Ohio. The derailed cars included seven tank cars carrying ethanol, one tank car carrying liquefied petroleum gas, and one tank car carrying phthalic anhydride. Twenty-six of the derailed cars were destroyed during the fire ensuing from the derailment. The NTSB determined that the probable cause of the derailment was a broken rail due to installation of an incorrect type of rail joint bar [6].
- On May 17, 2013, a passenger train derailed and was then struck by another passenger train travelling in the opposite direction on parallel track in Bridgeport, Connecticut. As a result of the collision, 73 passengers, two engineers, and a conductor were injured. Damage was estimated at \$18 million. Sections of the rail in the area of the derailment containing rail joint bars were removed and sent to the NTSB materials laboratory for further investigation which, to date, is ongoing.

In 2006, FRA amended the Track Safety Standards [7] in the Code of Federal Regulations (49 §213.119) by establishing requirements for railroads to: (1) conduct periodic inspections of joint bars depending on FRA track class and annual tonnage, and (2) collect data when cracked or broken joint bars are discovered in CWR territory. The data were documented in a Joint Bar Fracture Report, which was submitted to FRA Office of Safety. The intent of the reporting requirement was to study trends in the data to help determine the incipient conditions leading to joint bar failures. Specifically, the reporting requirements included the identification of: (1) cracked joint bars, (2) missing or loose fasteners, (3) conditions associated with wheel impact at joint (e.g. gap between rail ends and rail end batter), (4) proper rail anchoring, (5) adequate tie support, and (6) missing or loose bolts. Periodic inspections were shown to be more effective in finding defective joint bars than traditional track inspections. However, no definitive trends could be determined from studying the collected data.

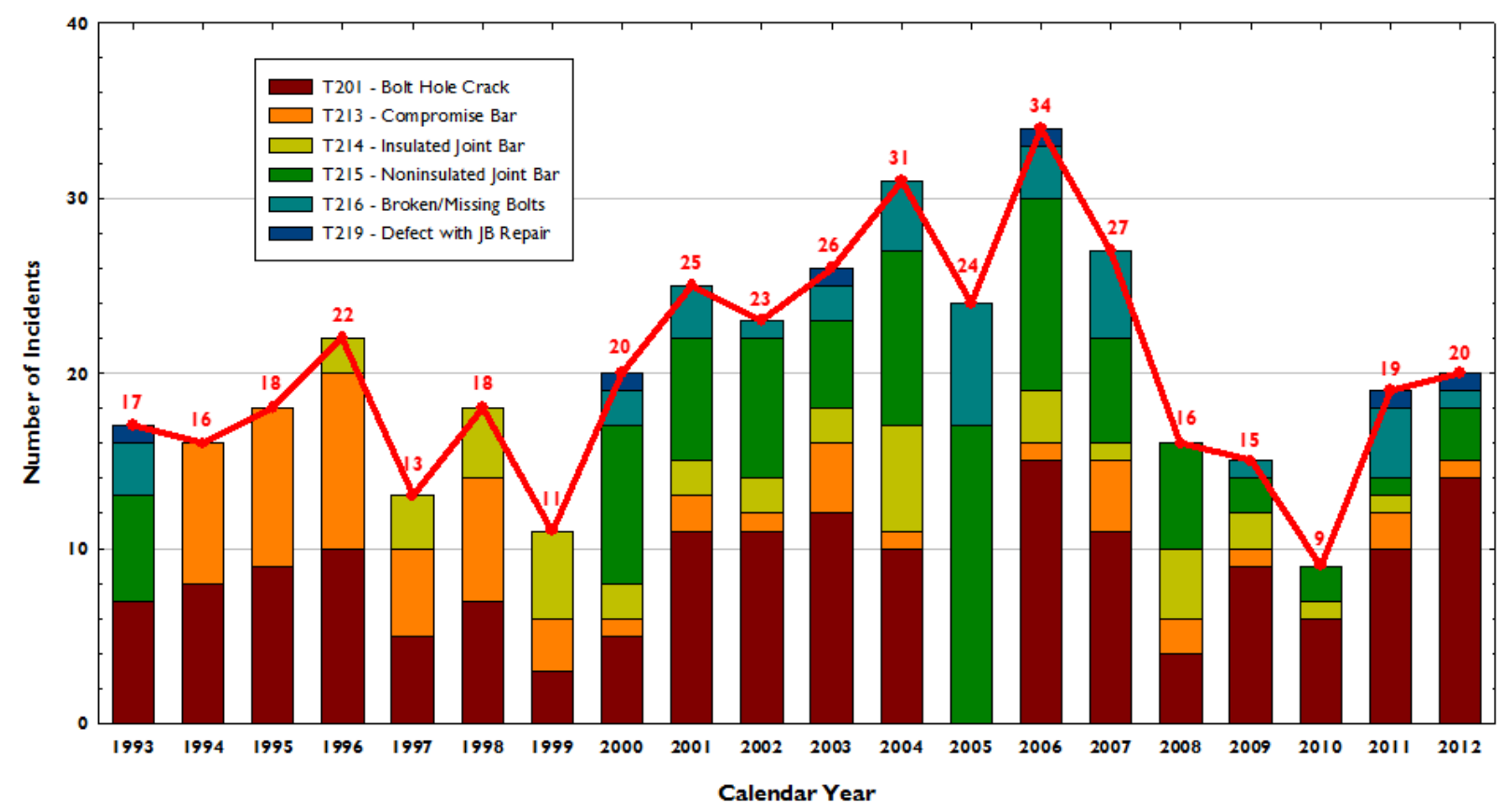

Figure 1: RAIRS Joint Failure-related Incidents, 1993-2012 
The FRA Office of Research and Development then initiated a research project to study the structural performance of rail joints and the associated track and operational factors leading to joint bar failure. Under contract to FRA, ENSCO, Inc. and Transportation Technology Center, Inc. (TTCI) made detailed field observations and measurements at various joint locations on revenue service track. Joint bars exhibiting indications of cracking and/or failure were discovered through automated inspections and traditional visual inspections. Randomly selected intact joint bar locations were also inspected. Automated inspections were carried out using the Optical Automated Joint Bar Inspection System (JBIS), developed by ENSCO under FRA sponsorship. Several railroads currently use the JBIS as part of their regular inspection programs.

\section{SUMMARY OF FIELD SURVEYS}

Through the cooperation of three Class I railroads, six field surveys were conducted on revenue service track in the United States between April 2012 and August 2013. During these field surveys, the survey team followed the Optical Automated JBIS vehicle in a hi-rail vehicle provided by the participating railroad. The team stopped at locations where a joint defect was identified by the JBIS inspection. Detailed information was collected and various measurements were recorded on the joint before the repair crews removed the defective joint bars.
Several of the joint bars identified as defective were collected and transported to TTCI facilities in Pueblo, Colorado for additional examinations and material testing.

The field survey team also stopped at randomly selected intact joint bars to collect the same information that was collected at the defective joint bar locations. The information collected from the intact joint bar locations established a control group to identify factors that might distinguish intact and failed joint locations as well as to gain insight into the general state of track conditions at temporary joints as compared to permanent joints in CWR territory.

Table 1 contains a brief summary of the six field surveys. For example, Survey 1 was conducted in April 2012 on a medium tonnage line in the western United States. During this first survey, a total of 23 joint locations were inspected over 75 miles of CWR territory. Of these 23 locations, three locations were determined to be defective. The six surveys covered a total of 581 miles, about 55 percent of which was CWR territory. A total of 203 joint locations were inspected, 58 locations (29\%) in CWR territory and 145 locations (71\%) in JR territory. Figure 2 shows the composition of the surveyed joint locations in terms of those in CWR and JR territory, and failed (or defective) versus intact (non-defective) locations. Data were also collected on 96 joint locations that did not contain a defect (which are referred to as "intact" joint locations); 53 locations in CWR and 43 joints in JR territory.

Table 1: Summary of Field Surveys on Rail Joints in Revenue Service

\begin{tabular}{|c|c|c|c|c|c|c|}
\hline & $1^{\text {st }}$ Survey & $2^{\text {nd }}$ Survey & $3^{\text {rd }}$ Survey & $4^{\text {th }}$ Survey & $5^{\text {th }}$ Survey & $6^{\text {th }}$ Survey \\
\hline Date & April 2012 & May 2012 & July 2012 & March 2013 & June 2013 & August 2013 \\
\hline Location & Western US & Midwest US & Midwest US & Southeast US & Midwest US & Midwest US \\
\hline Annual Tonnage $e^{1}$ & Medium & Low & Low & Medium & High & $\begin{array}{c}\text { Low (JR) } \\
\text { Medium (CWR) }\end{array}$ \\
\hline FRA Track Class & 4 & $\begin{array}{c}1 \& 2 \text { (JR) } \\
3 \text { (CWR) }\end{array}$ & $\begin{array}{c}3 \text { (JR) } \\
4 \text { (CWR) }\end{array}$ & 3 & 4 & $\begin{array}{c}\mathrm{X} \& 1 \text { (JR) } \\
1 \text { to } 4 \text { (CWR) }\end{array}$ \\
\hline Miles of CWR & 75 & 58 & 21 & 0.5 & 112 & 51 \\
\hline Miles of JR & 0 & 70 & 108 & 51.5 & 0 & 34 \\
\hline Total Miles Inspected & 75 & 128 & 129 & 52 & 112 & 85 \\
\hline Total Joint Locations & 24 & 47 & 48 & 36 & 26 & 22 \\
\hline No. of Defective Joint Locations & 3 & 31 & 34 & 26 & 0 & 13 \\
\hline No. of Defective Bars & 3 & 36 & 38 & 29 & 0 & 15 \\
\hline No. of Defects & 3 & 37 & 38 & 29 & 0 & 15 \\
\hline
\end{tabular}

\footnotetext{
${ }^{1}$ Annual tonnage is characterized as "Low” for less than 10 million gross tons (MGT) per year, "Medium” for 11 to 50 MGT per year, and "High” for greater than 50 MGT per year.
} 


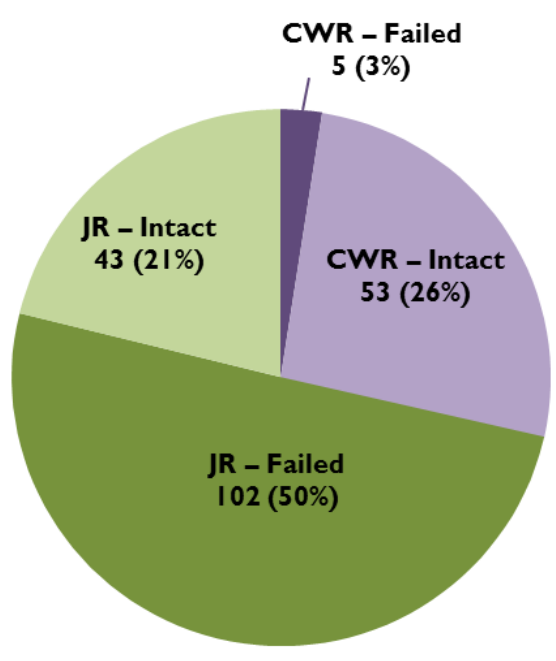

Figure 2: Composition of Surveyed Joint Locations (Total of 203 Joint Locations)

\section{OBSERVED FAILURE MODES}

Bars in the surveyed joint assemblies were identified as either a standard, compromise, or insulated bar. Compromise joint bars are designed to join rail sections of different sizes while keeping the gage and running surfaces in alignment. Insulated joint bars are needed where track circuits exist for signaling purposes. In addition, the surveyed bars were characterized as either angle, head-free, or head-contact bar (see Figure 3). Table 2 lists the number of failed or defective bars for these three different types found during the field surveys.

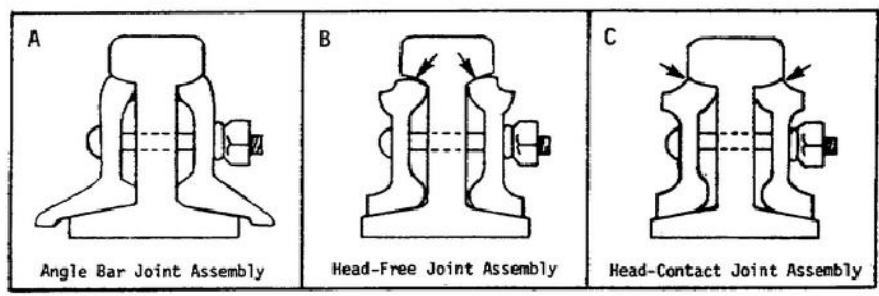

Figure 3: Types of Joint Assemblies

Table 2: Composition of Defective Bars in Field Surveys

\begin{tabular}{lc}
\hline Type & $\begin{array}{c}\text { Number of Defective Bars } \\
\text { (\% Total) }\end{array}$ \\
\hline Long-Toe Angle Bar & $47(39 \%)$ \\
Head-Free Joint Bar & $68(56 \%)$ \\
Head-Contact Joint Bar & $6(5 \%)$ \\
\hline TOTAL & 121 \\
\hline
\end{tabular}

The long-toe angle-bar design is usually associated with relatively light rail sizes (i.e. 90 and $100 \mathrm{lb}$ rail). These assemblies were not found in FRA Track Class 3 and above. Head-free bars were the most common design found during the surveys. When they are installed, head-free joint bars contact the rail at a single point in the head-web fillet region and the rail base. Head-contact joint bars are in full contact at the bottom of the rail head. Anecdotally head-contact joint bars are assumed to promote rail failures by head-web separation, but are also claimed to provide additional stiffening for better ride quality. The most common defect found in long-toe angle bars is referred to as a quarter crack, which originates at the corner of the spike hole in the bar (see Figure 4). Figure 5 is a photograph of a quarter crack that has grown through the entire cross section of the bar.

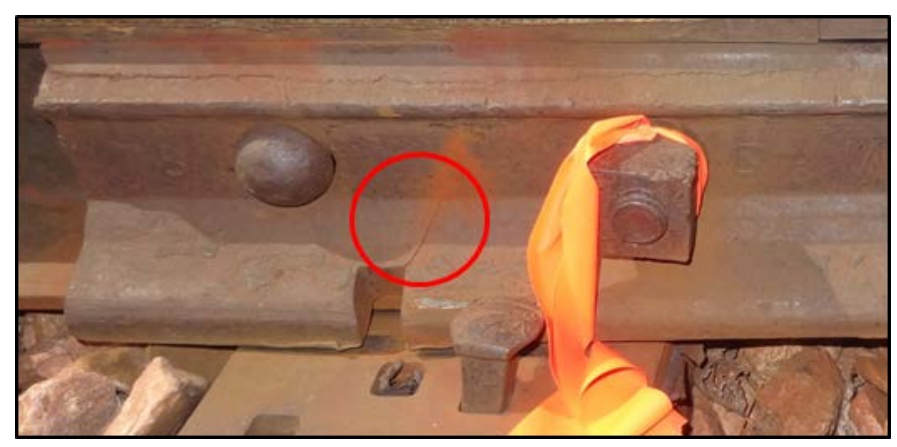

Figure 4: Quarter Crack

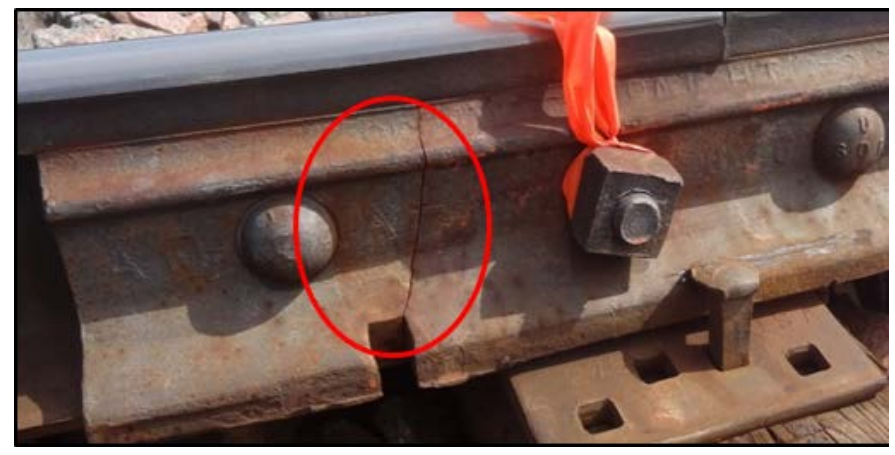

Figure 5: Quarter Break

The most common defect found in standard (head-contact and head-free) joint bars is a crack that originates from the top center of the bar. Figure 6 shows a photograph of the mating fracture surfaces of a top center crack in a standard joint bar. The macroscopically visible ridges, which may be referred to as clamshell or beach marks, are characteristic of metal fatigue. Evidence of plastic flow at the top of the bar can also be seen in the photograph. In some cases, fatigue cracks were seen to originate from the bottom center of a standard joint bar. For example, Figure 7 shows the fracture surface of an edge crack, which appears to emanate from the toe of the bar. 


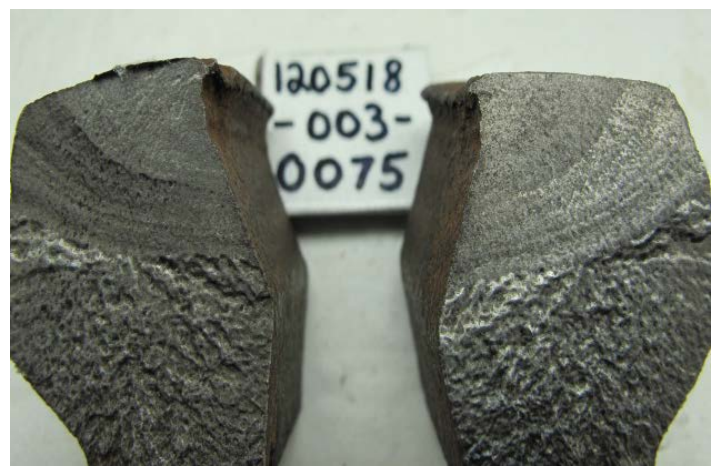

Figure 6: Top Center Crack

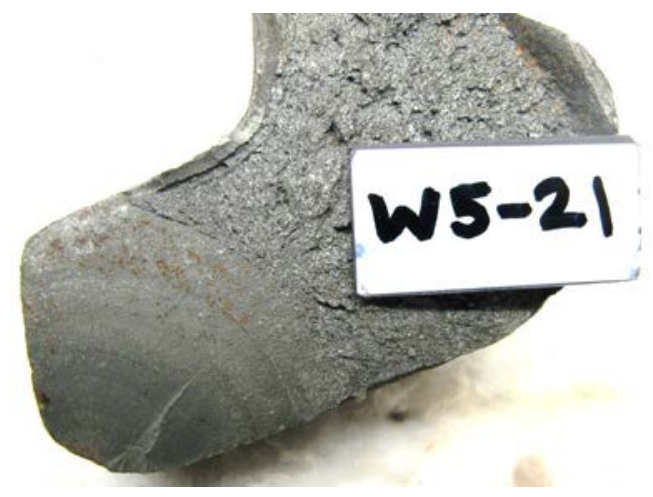

Figure 7: Bottom Crack in Standard Joint Bar

Figure 8 is a photograph of a crack emanating from a bolt hole. Bolt-hole cracks represented a small percentage (about $7 \%$ ) of the observed defects in the survey sample population. Upon further inspection of these types of defects, the origin of the cracking was observed to be a manufacturing anomaly, presumed to be nick from punching the hole in the bar.

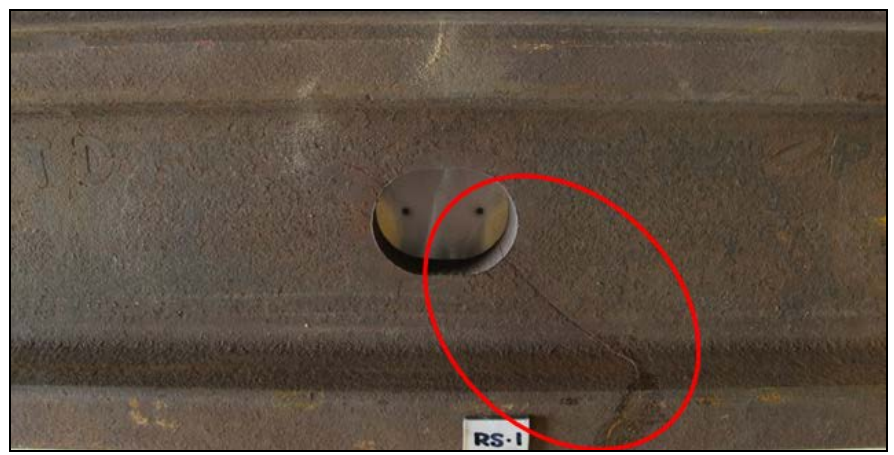

Figure 8: Bolt Hole Crack
A total of 122 defects were found in 121 failed or defective bars (i.e. one bar contained more than one defect) at 107 joint locations during the six field surveys. These defects were grouped into three types: (1) quarter defects, (2) center defects, and (3) bolt hole defects. Within each type, the defects were divided into sub-categories: either fully broken through or cracked. For quarter cracks and center cracks, the origin of the cracking, either from the top or the bottom of the bar, was documented. Table 3 lists the number of each different type of defect found during the field surveys in CWR and JR territory. Figure 9 is a graphical representation of the data in the table. A majority of the defects, 84 out of 122 (or 69 percent of the total), were center defects (either cracked or fully broken bars).

Table 3: Distribution of Defect Type in Field Surveys

\begin{tabular}{lccc}
\hline & $\begin{array}{c}\text { CWR } \\
\text { Territory }\end{array}$ & $\begin{array}{c}\text { JR } \\
\text { Territory }\end{array}$ & TOTAL \\
\hline Quarter Defects & 0 & 19 & 19 \\
- Full Break & 0 & 4 & 4 \\
- Top Crack & 0 & 6 & 6 \\
- Bottom Crack & & & \\
Center Defects & 0 & 19 & 19 \\
- Full Break & 5 & 53 & 58 \\
- Top Crack & 0 & 7 & 7 \\
- Bottom Crack & & & \\
Bolt Hole Defects & 0 & 4 & 3 \\
- Full Break & 0 & 5 & 5 \\
- Crack & 5 & 117 & 122 \\
\hline TOTAL & & & \\
\hline
\end{tabular}

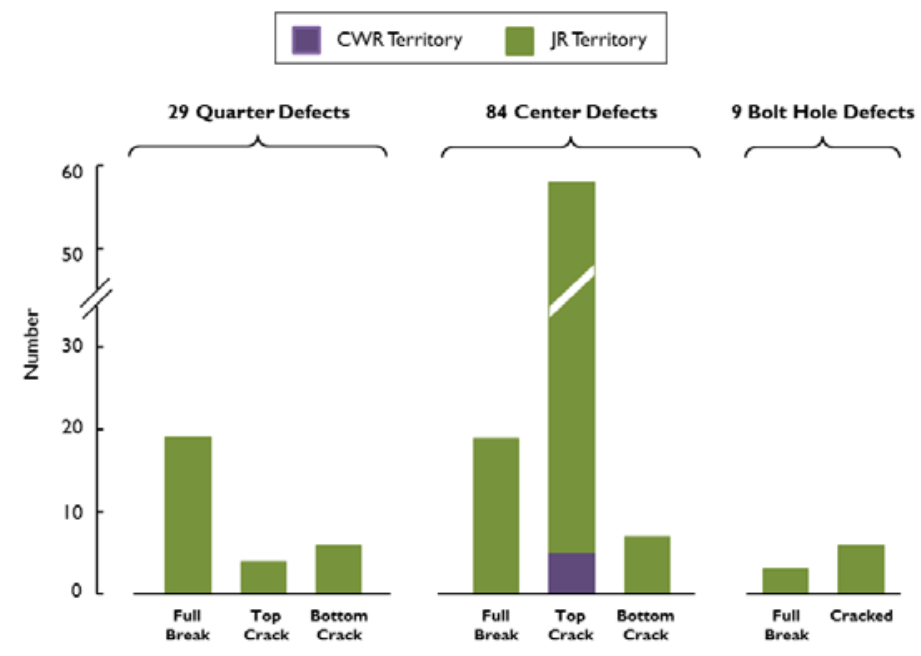

Figure 9: Defect Type Distribution in Field Surveys (Total of 122 Defects) 


\section{ANALYSIS OF COLLECTED DATA}

Data collected from the six field evaluation surveys were divided into two groups, one for CWR territory and another for jointed rail (JR) territory. A series of plots are presented in this section to compare various measurements at the surveyed joint locations. These plots summarize the collected information on: (1) joint movements in the vertical, lateral, and longitudinal direction; (2) geometry measurements for cross level, alinement, and profile; and (3) rail end batter. These plots include overlays of multiple measurements, which are intended to provide insight into possible relationships among these measurements and the documented conditions.

\section{Joint Locations in CWR Territory}

Data were collected from 58 joint locations in CWR territory during the six field evaluation surveys. The CWR joint locations were composed of: 39 intact standard joints, 14 intact insulated joints, 5 failed or defective joints, and 2 intact compromise joints. Moreover, all five defects were top center cracks in standard joint bars installed as temporary joints.

Figure 10 shows an overview of joint movements recorded at each of the joint locations in CWR territory. The horizontal axis in the plot is joint location sorted by increasing magnitude of vertical movement. The figure shows that the three failed joint locations have vertical movements of 0.375 inch or greater. The figure also shows that 12 joint locations were supported by concrete ties, which appear to have better support conditions than standard temporary joints. Similarly insulated joints appear to have better support conditions than standard temporary joints. However, it should be noted that several of the joints surveyed in CWR territory had been installed recently relative to the field evaluation date, and therefore did not have significant tonnage accumulation.

Figure 11 shows a similar overview of geometry measurements for cross level, alinement, and profile. In this figure, the horizontal axis is joint location sorted by ascending order of cross level under load. Three failed joint locations coincide with locations with adverse geometry conditions. The two other failed locations may be considered as statistical outliers; one was located at a switch where maintenance had recently been conducted relative to the survey date.

Figure 12 summarizes the rail end batter measurements in terms of rail end ramp and tread mismatch. Significant tread mismatch or abrupt ramps were measured at four of the five failed locations. A possible explanation may be due to the fact that failed joints in CWR territory are temporary joints where rail breaks or defects are replaced with rail plugs on which the head wear may not match up with the wear on the main rail.

\section{Joint Locations in JR Territory}

The field surveys were expanded to include jointed rail (JR) territory in order to increase the sample size for failed or defective joints. A total of 145 joint locations were surveyed in JR territory; 43 intact locations and 102 failed or defective locations. Failed or defective joint locations in JR territory were divided into two groups depending on the type of defects found in the bars. Table 4 lists the defect types for each category. The defects listed under the Type A defect location category typically originate from local stress concentration (e.g. at the corner or at a hole). Type B defect locations are made up of defects most likely to initiate from metal fatigue. The failed joint locations surveyed in JR territory comprise 24 Type A locations and 78 Type B locations.

Table 4: Categories for Defect Location Type

\begin{tabular}{|l|l|}
\hline Category & Bar Defects \\
\hline Type A & $\begin{array}{l}\text { - Full quarter break in long-toe angle bar } \\
\text { - Partial bottom quarter crack in long-toe angle bar } \\
\text { - All bolt hole cracks and breaks on all designs }\end{array}$ \\
\hline Type B & $\begin{array}{l}\text { - Full center break and all partial center cracks on } \\
\text { all bar designs } \\
\text { - Partial top quarter cracks on long-toe bars } \\
\end{array}$ \\
$\begin{array}{l}\text { - Full quarter breaks and all partial quarter cracks } \\
\text { on standard design bars }\end{array}$ \\
\hline
\end{tabular}

Figure 13 shows an overview of joint movements in JR territory. Failed or defective joint locations tend to have larger vertical movements than intact joint locations. This observation is consistent with joint movements measured in CWR territory. The figure also points out two repeated locations with failed or defective joints, which were identified from looking at records from the JBIS vehicle when it inspected the test zone prior to the field survey. Significant vertical movements were measured at both locations with repeated failures.

An overview of the geometry measurements in JR territory is shown in Figure 14. Failed or defective joint locations have a significantly larger cross level under load and profile under load than the intact joint locations. The two joint locations with repeated failures are annotated in the figure.

A subset of the data collected on joints in JR territory was gathered to extract only joint locations in FRA Track Class 3. This subset was used to examine the possible effect of rail end batter on joint bar failure on main lines with relatively high tonnage and operating speeds. This subset consisted of 84 joint locations; 24 intact joints and 60 failed or defective joints. The horizontal axis in Figure 15 is joint location sorted by rail end batter height in ascending order. Positive values on the vertical axis refer to the left rail end batter, and negative values are the right rail end batter, which are reasonably consistent for the sample population. Moreover, the figure shows moderate to significant levels of rail end batter at failed or defective joint locations. The figure also shows that most of the rail end batter measurements are less than industry recommended limits. However, these trends with rail end batter at failed locations compared to intact locations were not observed on lower track classes (implying lower train speed) with low tonnages. That is, the measurements of rail end batter between failed and intact locations were not significantly different on lower track classes in JR territory. 


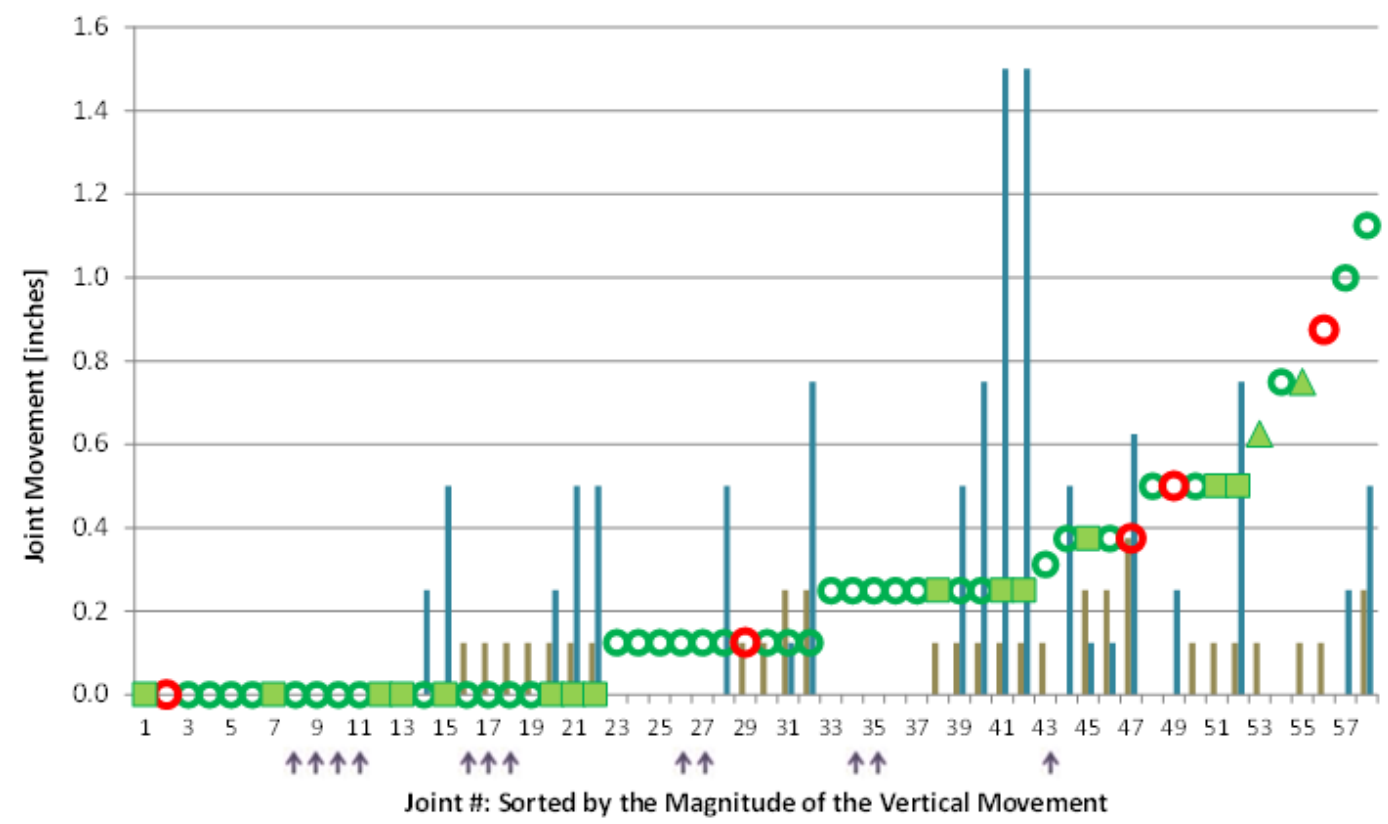

n Lateral Movement

- Longitudinal Movement

Vertical Movement for: - INTACT STANDARD JOINT - failed STANDARD JoInt - INTACT INSULATED JOINT $\triangle$ INTACT COMPROMISE JOINT

Figure 10: Overview of Joint Movements in CWR Territory

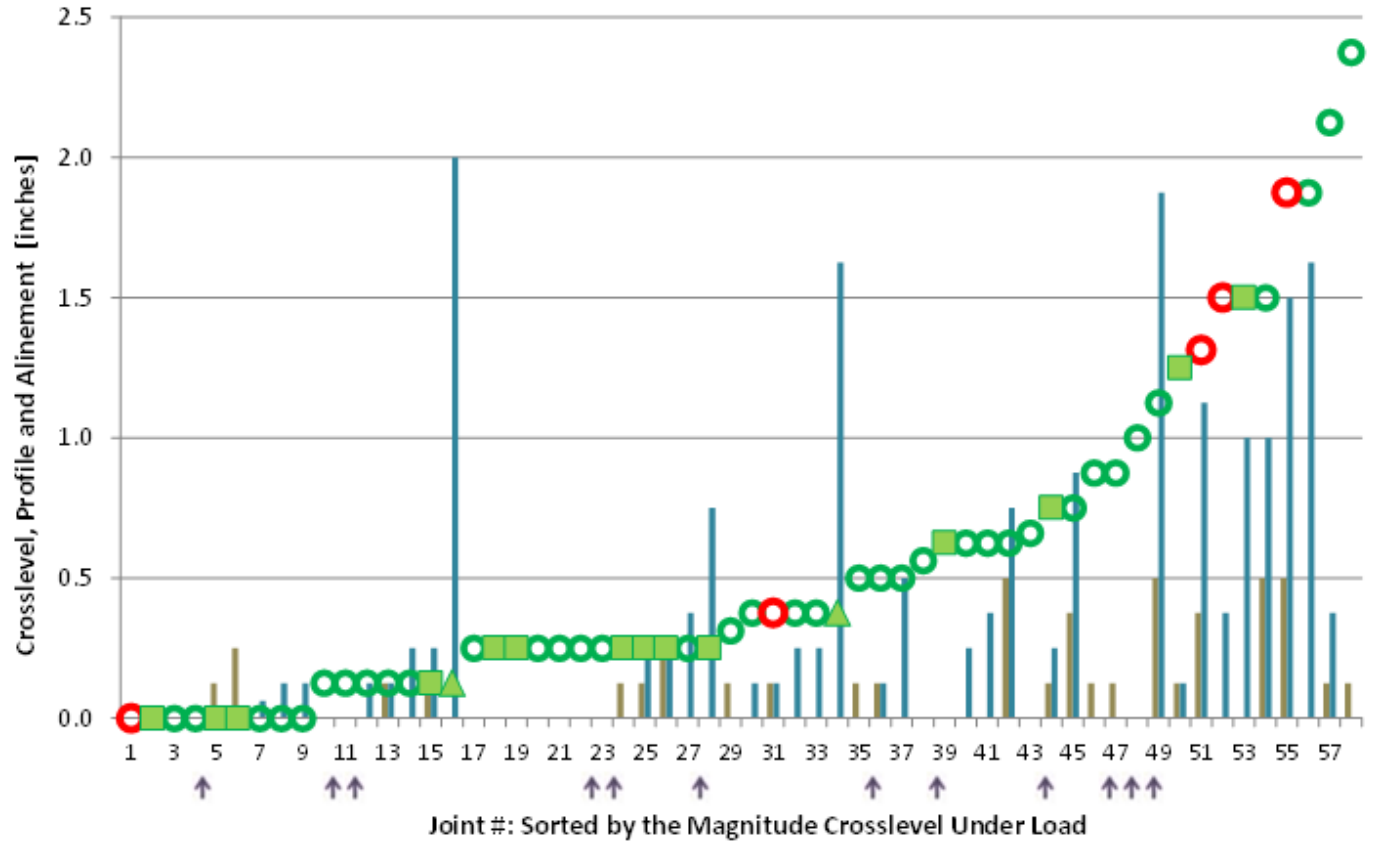

alinement

n Profile Under Load Crosslevel Under Load for: OINTACT STANDARD JOINT - falled standard joint - INTACT INSULATED JOINT $\triangle$ INTACT COMPROMISE JOINT

Figure 11: Overview of Geometry Measurements in CWR Territory 


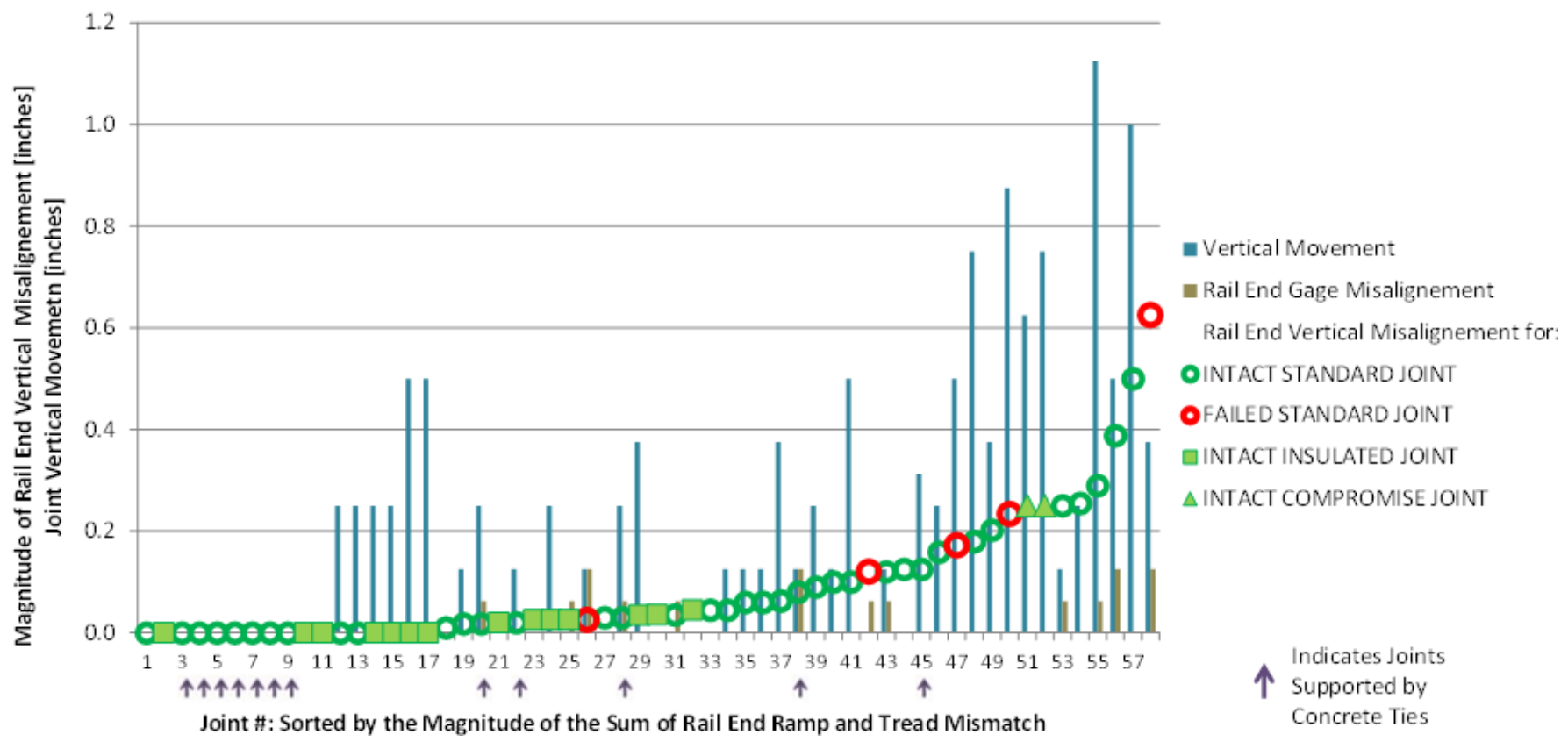

Figure 12: Overview of Rail End Batter in CWR Territory

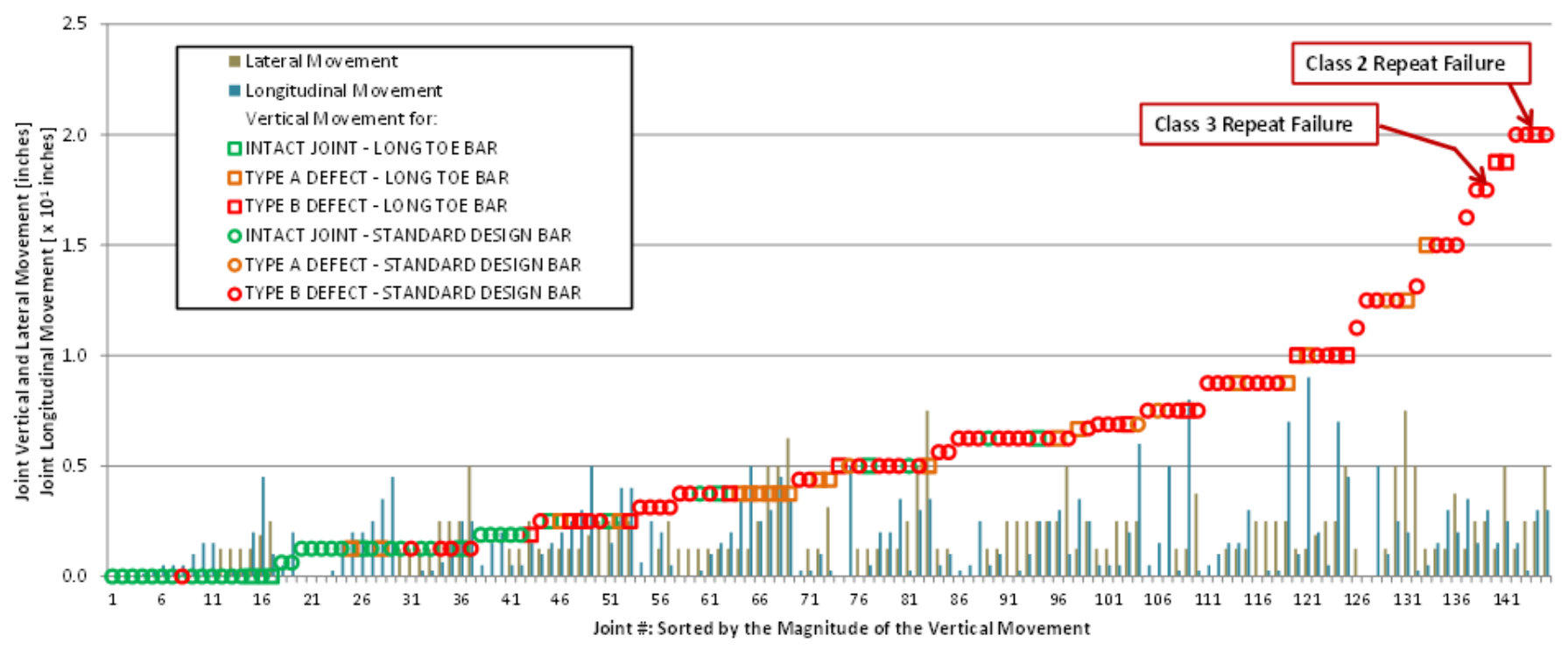

Figure 13: Overview of Joint Movements in JR Territory 


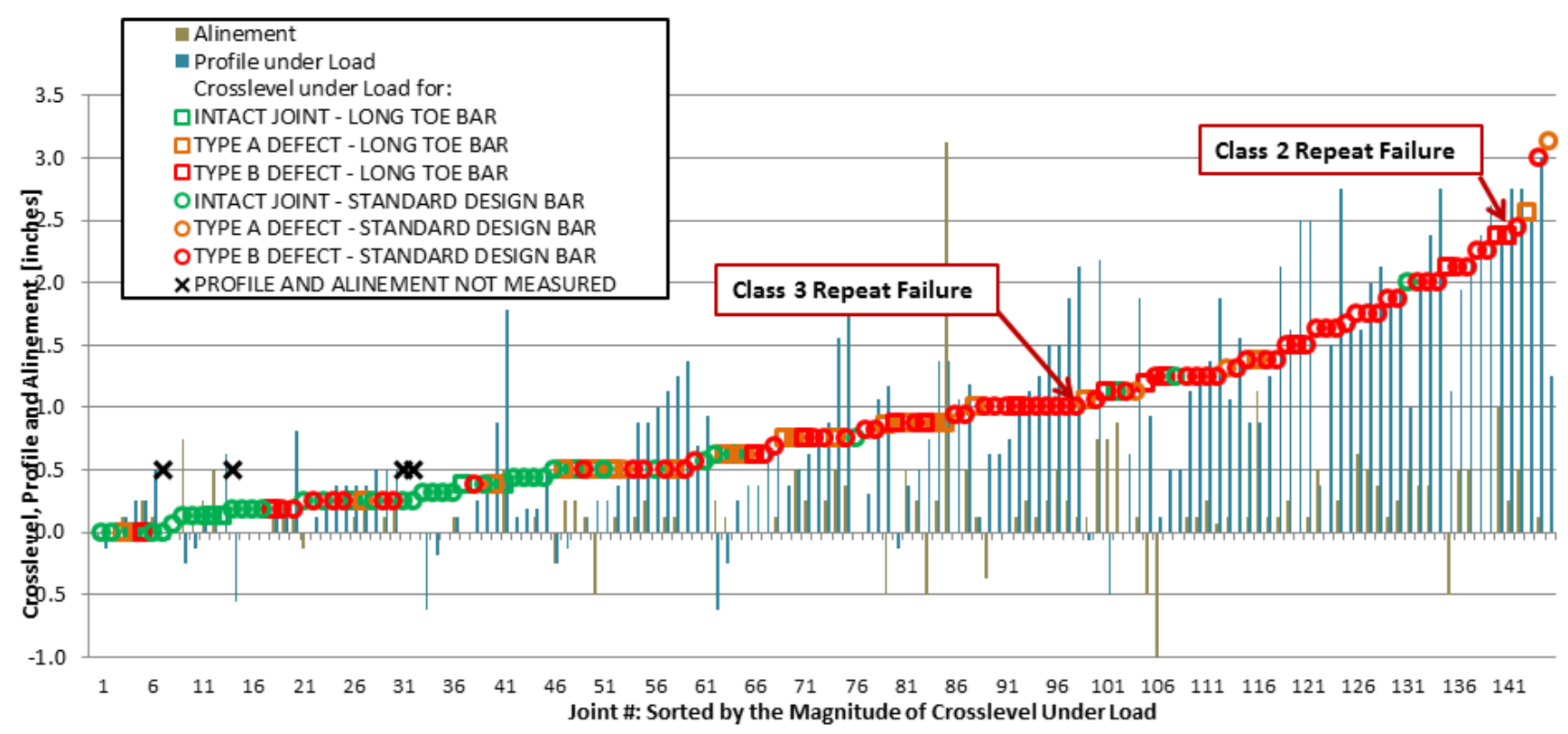

Figure 14: Overview of Geometry Measurements in JR Territory

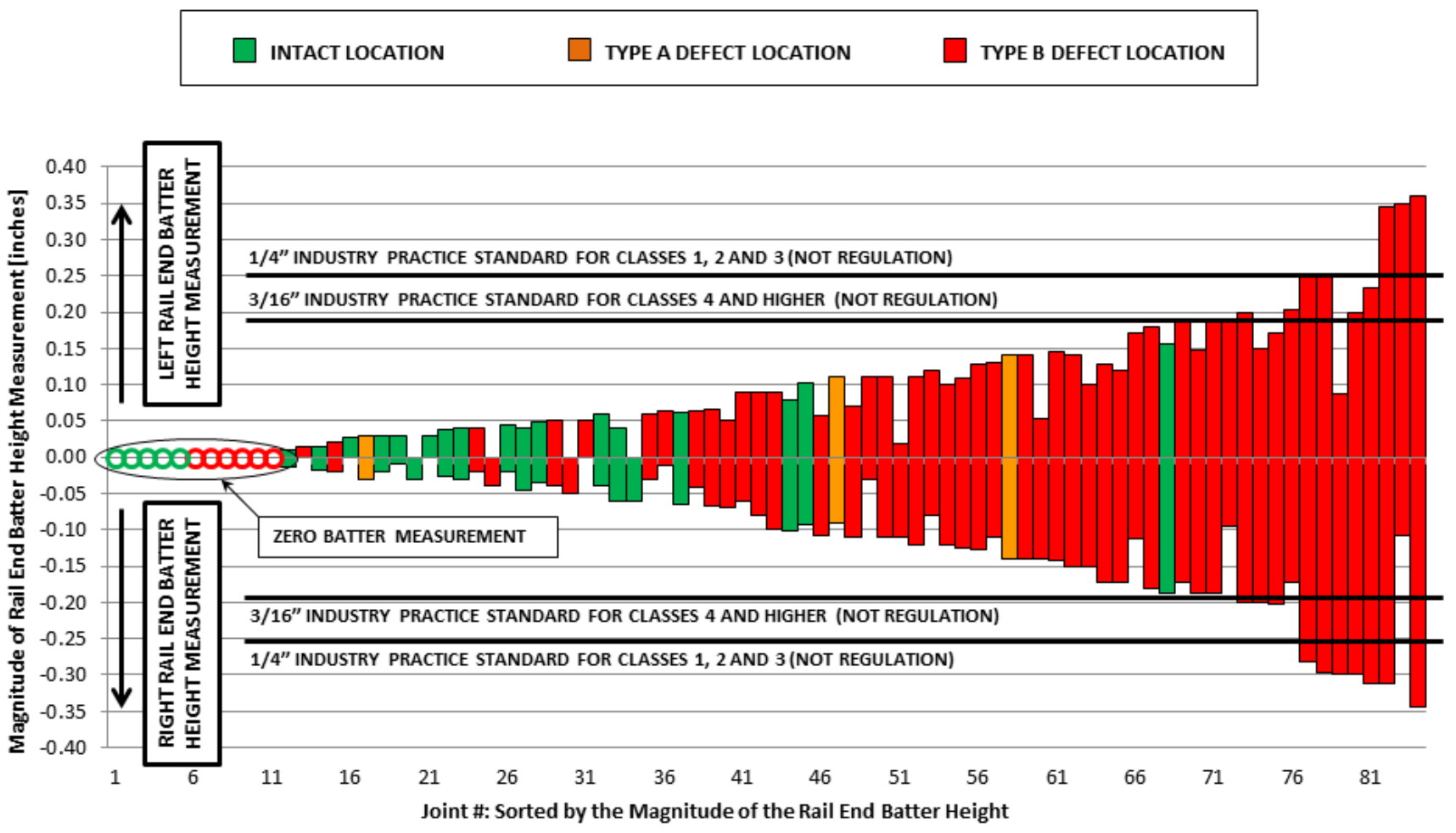

Figure 15: Overview of Rail End Batter in Class 3 JR Territory 
The results presented in this paper are a glimpse of the overall analyses conducted to study the collected survey data. Additional details and results of the first three surveys are described and presented in a technical report [8] prepared by ENSCO.

\section{DISCUSSION}

Based on the observations from the field surveys and previous experience, relatively large deflections can occur at a rail joint as wheels pass over it. These larger deflections may be attributed to the reduced section properties (i.e. crosssectional area and area moments of inertia) of the joint bars compared to those of a continuous rail. However, another factor contributing to large deflections might be degraded tieballast support. For example, the apparent correlation between vertical joint movement and failed joint locations suggests that tie-ballast support condition plays a role in mechanical performance of rail joints. However, the causes and the effects of joint deflection and tie-ballast degradation are difficult to distinguish because large deflections can lead to and may accelerate track degradation, which in turn can affect the structural performance of the joint. When a single wheel passes over the joint, the rail ends deflect downward one at a time, creating a small step and mismatch in rail heights. The size of the step varies depending on the original gap distance between rail ends. The step causes wear from repeated wheel passes, which is referred to as rail end batter. The structural performance of the rail joint also degrades as the bolts and the supporting ballast loosen. Eventually, increased deflections and joint anomalies (e.g. excessive gap between rail ends, end batter, loose or missing bolts, etc.) induce dynamic amplification of wheel loads at the joint, which accelerates wear. This viscous cycle of deteriorating track and joint conditions leading to and caused by high dynamic wheel impact loads is shown schematically in Figure 16. Failure of the joint (i.e. broken bar or rail) can occur anywhere within the cycle.

The mechanics of bolted rail joints in terms of structural performance under applied loads is being examined through computational analysis. Specifically, finite element analyses (FEA) are under development to help interpret the trends observed in field survey data. Moreover, FEA of bolted rail joints is the topic of the second paper in this two-part series [9].

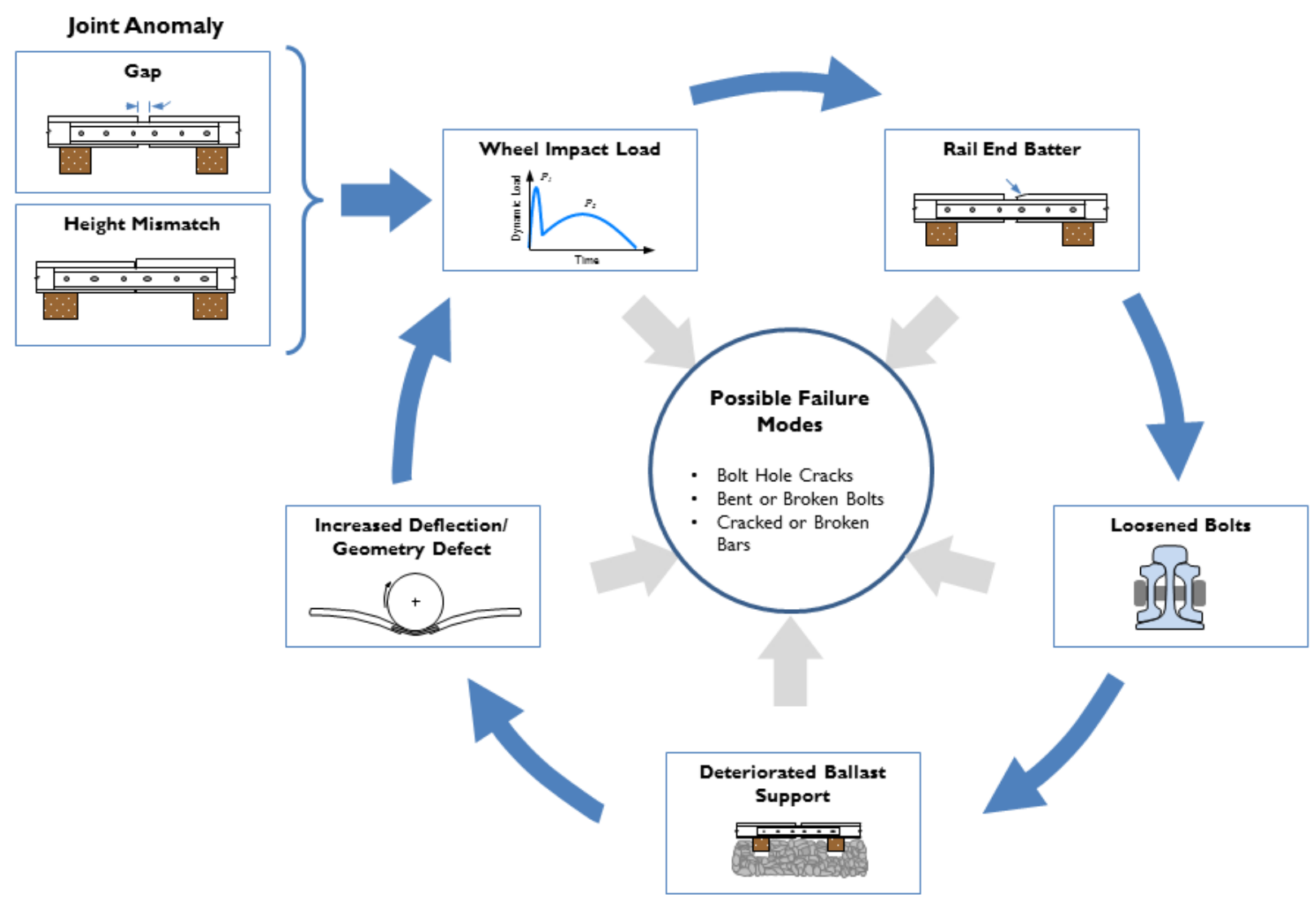

Figure 16: Cycle of Joint and Track Degradation Interacting with Wheel Impact Loads 


\section{CONCLUDING REMARKS}

Under a research project sponsored by the FRA Office of Research and Development, a detailed methodology for collection of field data from joint bar inspections was developed and refined. Six field evaluation surveys were conducted between April 2012 and August 2013 by a survey team consisting of ENSCO and TTCI personnel in close cooperation with three participating railroads. Data were collected from a total of 203 joint locations, 58 joints in CWR territory and 145 joints in JR territory. A total of 122 defects were found in 121 defective bars at 107 joints during these six field surveys.

The observations of the joints inspected on CWR territory are summarized as follows:

- The maintenance of track conditions such as vertical movement and track geometry at permanent (i.e. insulated joints) was found to be significantly better than temporary joints which were intended to be welded.

- Neutral temperature measurements were not conducted during the field surveys. However, indirect signs of changes in rail thermal stress were recorded, such as longitudinal rail and joint bar movements.

The observations of joints in JR territory are summarized as follows:

- Joint vertical movement, cross level under load, and profile under load emerge as factors affecting joint bar failure rates.

- Longitudinal movement does not appear to be a significant factor.

Additional surveys are being planned for future work. These surveys will be conducted on CWR and jointed territory in which historical data indicates a high likelihood of joint failures. In addition, selection of the test zones will be such that

- At least one zone on jointed territory surveyed previously

- At least one zone on CWR territory surveyed previously.

Meanwhile finite element analyses will continue to examine the mechanical performance of rail joints under applied loading. These computational analyses may help better understand the trends observed in the data collected during the field evaluation surveys.

\section{ACKNOWLEDGMENTS}

The work described in this paper was sponsored by the Office of Research and Development, Federal Railroad Administration (FRA), U.S. Department of Transportation, under the direction of Mr. Gary Carr, Chief of the Track Research Division. The authors also acknowledge the contributions of Mr. Muhammad Akhtar, formerly of the
Transportation Technology Center, Inc. (now with Parsons Brinckerhoff) who provided technical support in the conduct of the field surveys.

The cooperation of three Class I railroads in the US is gratefully acknowledged. This work would not have been possible without the railroads' participation and collaboration.

\section{REFERENCES}

1. Jeong, D.Y., "Progress in Rail Integrity Research,” Volpe Center Final Report to Federal Railroad Administration, DOT/FRA/ORD-01/18, October 2001.

http://www.volpe.dot.gov/coi/pis/docs/fail/progress.pdf

2. Federal Railroad Administration, Railroad Accident/ Incident Reporting System (RAIRS).

http://safetydata.fra.dot.gov/officeofsafety/default.aspx

3. National Transportation Safety Board, "Derailment of Union Pacific Railroad Train QFPLI-26 at Eunice, Louisiana, May 27, 2000,” Railroad Accident Report NTSB/RAR-02/03, PB2002-916303, April 2002. http://www.ntsb.gov/doclib/reports/2002/RAR0203.pdf

4. National Transportation Safety Board, "Derailment of Canadian Pacific Railway Freight Train 292-16 and Subsequent Release of Anhydrous Ammonia Near Minot, North Dakota, January 18, 2002,” Railroad Accident Report NTSB/RAR-04/01, PB2004-916301, March 2004. http://www.ntsb.gov/doclib/reports/2004/RAR0401.pdf

5. National Transportation Safety Board, Railroad Accident Brief, Accident No. DCA-05-FR-002, Pico Rivera, California, October 16, 2004, NTSB/RAB-05/02, Adopted May 31, 2005.

http://www.ntsb.gov/doclib/reports/2005/RAB0502.pdf

6. National Transportation Safety Board, Railroad Accident Brief, Accident No. DCA-08-FR-001, Painesville, Ohio, October 10, 2007, NTSB/RAB-09/02, Adopted June 1, 2009.

http://www.ntsb.gov/doclib/reports/2009/RAB0902.pdf

7. Federal Register, Vol. 71, No. 196, Track Safety Standards; Inspection of Joints in Continuous Welded Rail (CWR), Docket No. FRA 2005-22522, October 11, 2006. http://www.fra.dot.gov/eLib/details/L03219

8. Bruzek, R., Jamieson, D., "Field Activities for Joint Bar Failure Study, Phase 1,” ENSCO Final Report to Federal Railroad Administration, ENGR-REPT-0000136, September 2012.

9. Carolan, M.E., Jeong, D.Y., Perlman, A.B., "Engineering Studies of Joint Bar Integrity, Part II: Finite Element Analyses,” Proceedings of the 2014 Joint Rail Conference, Colorado Springs, CO, JRC2014-3708, April 2014. 